\title{
Optimum similarity analysis with applications to elastohydrodynamic lubrication
}

\author{
H. Moes \\ University of Twente, Department of Mechanical Engineering, Tribology Group, P.O. Box 217, 7500 AE Enschede (Netherlands)
}

(Received October 15, 1991; accepted January 14, 1992)

\begin{abstract}
A new method for deriving similarity solutions is introduced. The application proves to be very simple and straightforward. For any set of analytical relations, whether dimensionally homogeneous or not, the greatest possible reduction in the number of quantitics may casily be accomplished. The method is introduced by means of simple applications and the results are compared with the results of conventional dimensional analysis.

In elastohydrodynamic lubrication in particular, this method may readily be accepted as a very helpful tool when dealing with the extensive sets of analytical relations involved. Yet it is certainly not restricted to lubrication problems only.

Furthermore, the results show that physical properties seem to have no place in similarity analysis. Similarity analysis is pure string manipulation. Consequently, the method has proved to be perfectly suitable for personal computer applications.
\end{abstract}

\section{Introduction}

\subsection{Similarity analysis}

A model in mathematical analysis based on a physical phenomenon may be stated as a set of relations such as algebraic equations, differential equations, integral equations etc. It will in general be restated by applying a reduced number of quantities. This has to be performed by introducing well-chosen functions of the original quantities. Often some of these substitutions are quite obvious, e.g. simple additions and subtractions, and they will be performed almost automatically. The selection of a set of power products of quantities (henceforth PPs) which will lead to a maximum reduction in the number of original quantities, though, is far from easy.

Similarity analysis is the name given to the various techniques applied to yield a workable set of PPs [1]. Dimensional analysis is the most well-known technique among them [2]. A modification called vectorial dimensional analysis was introduced later $[3,4]$. The problems related to the application of vectorial dimensional analysis raise doubts about the introduction of typical physical concepts such as dimensions into the mathematical analysis and this has provided the impetus for the present work.

To begin with, let us assume that a set of relations in mathematical analysis involves $m$ original quantities $Q_{i}$, and let us also assume that a set of $n$ PPs for similarity $\pi_{i}$ has been derived for this set of relations. Then $\pi_{i}$ can be defined by the following PPs:

$\pi_{i} \equiv \gamma_{i=1}^{m} Q_{j}^{\alpha_{i j}} \quad$ for $i=1,2, \ldots, n \quad$ and $n<m$

with $\gamma_{i}$ and in particular $\alpha_{i j}$ representing real numbers. Actually $\gamma_{i}$ is quite arbitrary. For reasons of simplicity, therefore, we will assume $\gamma_{i}=1$ throughout this paper.

The first author who systematically derived PPs for similarity was Vaschy [5] in 1892. Since these PPs were essentially non-dimensional, they became well known by the name of "dimensionless numbers". Later, Buckingham $[6,7]$ introduced the symbol $\pi_{i}$ applied above.

As we shall see later, when deriving PPs for similarity it will be more convenient to indicate $\pi_{i}$ by the dashed quantity $\bar{Q}_{i} ; Q_{i}$ represents a quantity appearing with unit power and is explicitly in the PP for similarity $\bar{Q}_{i}$; i.e.

$\bar{Q}_{i} \equiv Q_{i} \prod_{j=n+1}^{m} Q_{j}^{\alpha{ }^{a j}} \quad$ for $i=1,2, \ldots, n \quad$ and $n<m$

We will call this a set of similarity quantities (henceforth SQs). Later these SQs may be arranged in a new set of linearly independent PPs in order to derive a suitable set of PPs for similarity $\pi_{i}$. This is a matter of personal choice, though, and therefore will not be discussed here.

It will also prove convenient to discriminate between the following five types of SQs: 
(1) control similarity parameters;

(2) dependent similarity parameters;

(3) independent similarity variables;

(4) integration similarity variables;

(5) dependent similarity variables.

These SQs will be defined as follows. On the one hand, the quantities appearing in an SQ may all be parameters. We will call this parametric $S Q$ a similarity parameter. Moreover, this type of similarity parameter may be treated as a control similarity parameter or as a dependent similarity parameter. This distinction is not exact, though. It has to be decided from the problem definition under consideration. It is not crucial, anyhow.

On the other hand, variables too may appear in an SQ. We will call this variable $S Q$ a similarity variable. If all the variables in a similarity variable are independent then we will call it an independent similarity variable. However, in integral expressions an extra integration similarity variable will often be needed.

Finally, if at least one of the variables in a similarity variable is dependent then we will call it a dependent similarity variable.

\subsection{The meaning of similarity analysis}

At this stage it should be mentioned that the advantages obtaincd by applying similarity analysis are quite often not fully understood. In brief, similarity analysis is a very useful tool, since, for example:

(i) Model experiments are based on similarity parameters [8].

(ii) Analogues are based on identical formulations for the relations between sets of similarity quantities in different domains of science, e.g. the analogues based on electricity which led to the analogue computer.

(iii) Simplifications that may be quite substantial follow from introducing similarity quantities. Sometimes, partial differential equations reduce to ordinary differential equations, or even to algebraic equations [9].

(iv) The reduction in the number of quantities needed may lead to substantial savings in both time and money when carrying out numerical calculations [9].

(v) A better presentation of the solution of a problem can be given, since only a limited number of graphs, tables etc. will be needed [9].

(vi) Curve fitting is facilitated, as in the application to elastohydrodynamic lubrication in Section 4.2.

Considering these six characteristics it is remarkable to see the haphazard applications of similarity analysis in engineering. There often seems to have been more use of intuition and diligence than of pure reasoning. Consequently, the smallest possible set of SQs has quite often not been attained (see, for instance, ref. 10 for a discussion on the application to elastohydrodynamic lubrication).
The situation is much more favourable in hydrodynamics. For instance, Schlichting [9] employs an impressive collection of applications of boundary-layer theory, all of them seemingly appearing out of the blue. By the way, one of these applications, the application to the well-known problem of Blasius, served as a perfect exercise when testing the new method to be presented.

This is an exception, though. The main reason for this quite unsatisfactory situation may be that the wellknown dimensional analysis, being both straightforward and simple, but generally leading to at least one redundant dimensionless number, offers a very attractive tool and consequently is very generally applied. However, the application of vectorial dimensional analysis [4], which may more frequently lead to optimal similarity, has only been applied when the quite mysterious vectorial components of the spatial dimensions for the derived quantities could be guessed, and unfortunately the quite useful inspectional analysis [11] is just a method of verification.

The new method to be presented in this paper is both simple and straightforward and nevertheless will invariably lead to an optimum similarity solution.

\section{A simple model: Jeffreys' equation [12]}

This method, called optimum similarity analysis, is based on completely new concepts and therefore will be introduced step by step by means of four successivc procedures, all applied to one and the same formula. The first step will be a quite unsatisfactory analysis. The following steps will gradually lead to improvements until the fourth and final step brings about the optimum similarity that has been aimed for.

For demonstration purposes a very suitable mathematical formulation happens to be the equation according to Jeffreys [12] for the drainage of a vertical plate, reading

$\frac{\partial h}{\partial t}+\frac{\rho g}{\mu} h^{2} \frac{\partial h}{\partial x}=0$

where $h$ represents the thickness of the fluid layer, $\rho$ its density and $\mu$ its viscosity; $g$ represents the acceleration due to gravity, $x$ the coordinate along the plate and $t$ the time; see also Fig. 1 .

To begin with, this equation will be normalized in the conventional way, i.e. both Lagrange's solution applying integral curves and Cauchy's method are purposely neglected. The boundary conditions will be discussed later. 


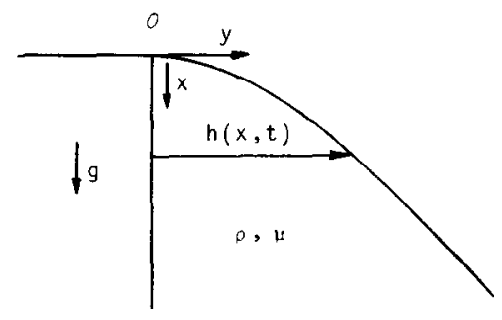

Fig. 1. The drainage of a vertical plate after Jeffreys.

\subsection{Normalization}

Normalization of an equation means replacing the original variables by normalized variables, i.e. the original variables $h, x$ and $t$ divided by the respective characteristic parameters $\hat{h}, \hat{x}$ and $\hat{t}$. A method for obtaining these parameters will be discussed later. It will become the key to the new method. For the time being, however, we define

$\bar{h} \equiv \frac{h}{\hat{h}} \quad \bar{x} \equiv \frac{x}{\hat{x}} \quad \bar{t} \equiv \frac{t}{\hat{t}}$

Thus Jeffreys' equation, introduced above as eqn. (2), may be replaced by

$\frac{\partial \bar{h}}{\partial \bar{t}}+\pi_{1} \bar{h}^{2} \frac{\partial \bar{h}}{\partial \bar{x}}=0$

where

$\pi_{1} \equiv \frac{\rho g \hat{h}^{2} \hat{t}}{\mu \hat{x}}$

$\pi_{1}$ represents a PP which is dimensionless but of little use because of the as yet unknown three characteristic parameters involved.

The next step in this series of procedures, therefore, will be a method to derive these characteristic parameters.

\subsection{A provisional similarity solution}

Substitution of the definitions in eqn. (3) into eqn. (5) leads to

$\frac{\bar{h}^{2} \bar{t}}{\bar{x}}=\pi_{1}-1 \frac{\rho g h^{2} t}{\mu x}$

By substituting $\pi_{1}=1$ into cqn. (6) (this is allowed since the three characteristic parameters have not been chosen yet) there remains

$\frac{\bar{h}^{2} \bar{t}}{\bar{x}}=\frac{\rho g h^{2} t}{\mu x}$

One of the solutions to eqn. (7) reads

$$
\bar{h} \equiv h\left(\frac{\rho^{2} g}{\mu^{2}}\right)^{1 / 3} \quad \bar{x} \equiv x\left(\frac{\rho^{2} g}{\mu^{2}}\right)^{1 / 3} \quad \bar{t} \equiv t\left(\frac{\rho^{2}}{\mu}\right)^{1 / 3}
$$

Consequently Jeffreys' equation may be replaced by the similarity solution

$\frac{\partial \bar{h}}{\partial \bar{t}}+\bar{h}^{2} \frac{\partial \bar{h}}{\partial \bar{x}}=0$

As the embarrassing characteristic parameters have been eliminated this is a substantially improved relation compared with eqn. (4). It may even seem to be quite useful. Still, this is just the beginning.

\subsection{Dimensional analysis}

Next, a simple version of the Vaschy-Buckingham approach [5-7] will be applied to this problem. The four quantities (or PPs of quantities) that may be distinguished in Jeffreys' equation are $h, x, t$ and $\rho g /$ $\mu$. Between these quantities there are two independent dimensional relations, i.e. through length and through time. Consequently the following dimensionless numbers may be derived (see Table 1).

$\bar{h} \equiv h \frac{t \rho g}{\mu} \quad \bar{x} \equiv x \frac{t \rho g}{\mu}$

Thus Jeffreys' equation may be replaced by the ordinary differential equation (see also Appendix A)

$\frac{\mathrm{d} \bar{h}}{\mathrm{~d} \bar{x}}=\frac{\bar{h}}{\bar{h}^{2}+\bar{x}}$

which can readily be solved (substituting $\bar{h}=z(\bar{x})^{1 / 2}$ ):

$\bar{h}=\left(c^{2}+\bar{x}\right)^{1 / 2}-c$

The integration constant $c$ still has to be estimated by means of the uniqueness condition that applies; i.e. an initial condition or a boundary condition.

\subsection{Optimum similarity analysis}

The reader may have noticed that the dimensionless numbers in eqns. (8) may also follow from eqn. (7) by simply introducing $\bar{x}$ for the product $\bar{x} \bar{t}$ and $\bar{h}$ for $\bar{h} \bar{t}$, thus eliminating $\bar{t}$. This automatically raises the question as to what would be the result of introducing $\bar{h}$ for $\bar{h}\left(\bar{t}(\bar{x})^{1 / 2}\right.$ and eliminating both $\bar{t}$ and $\bar{x}$. According to eqn. (8) this would simply lead to

$\bar{h} \equiv h\left(\frac{\rho g t}{\mu x}\right)^{1 / 2}$

TABLE 1. Matrix of coefficients for the dimensional formulae for Jeffreys' model

\begin{tabular}{lrc}
\hline & $L$ & $T$ \\
\hline$h$ & 1 & 0 \\
$x$ & 1 & 0 \\
$t$ & 0 & 1 \\
$g g / \mu$ & -1 & -1 \\
\hline
\end{tabular}


which eventually reduces Jeffreys' equation to the algebraic equation (see also Appendix A)

$\overline{h^{3}}-\bar{h}=0$

This has the immediate solutions

$\bar{h}=0, \pm 1$

Indeed, $\bar{h}=1$ is a particular solution to Jeffreys' problem. This unquestionably represents the optimum similarity solution, for, notwithstanding the lack of uniqueness conditions, the original partial differential equation has been solved. Of course this implies that some kind of uniqueness condition has been introduced; this will be discussed in Section 3.

\subsection{Discussion}

Referring to these results for just one equation we may draw some conclusions in relation to optimum similarity solutions in general. However, since singleequation models are a rarity, these conclusions will be quite provisional in character.

The unique SQ for Jeffreys' equation stems from the definition of the PP for similarity $\pi_{1}$ that has been derived by applying a primitive normalization procedure. Hence this normalization procedure can be considered as the first step in the derivation of an optimum similarity solution. The second and final step is the substitution of the respective variables for the characteristic parameters, which is equivalent to omitting the caps in the definition of $\pi_{1}$.

For a multitude of $\pi_{i}$ values with $i=1, \ldots, n$, an intermediate step might have been needed, i.e. the derivation of $n$ new $\pi_{i}$ values with explicit occurrence of each parameter by arranging these $\pi_{i}$ values in $n$ PPs. For this simple model, though, $\pi_{1}$ leads to just one SQ. Anyhow, the number of SQs (or $\bar{Q}_{i}$ values; see eqn. (1)) in an optimum similarity solution quite simply equals the number of PPs (or $\pi_{i}$ values) of quantities as acquired by primitive normalization.

\section{More complicated modelling}

\subsection{A modification of Jeffreys' model}

An interesting problem that still remains to be solved is the role of the uniqueness conditions. First, it may be noticed that the optimum similarity solution according to eqn. (12) includes one of the following three pairs of uniqueness conditions:

for $\vec{h}=0 \quad$ if $x=0$ then $h=0 \quad$ and if $t=0$ then $h=0$

for $\bar{h}=-1 \quad$ if $x=0$ then $h=0 \quad$ and if $t=0$ then $h=-\infty$

for $\bar{h}=1 \quad$ if $x=0$ then $h=0$ and if $t=0$ then $h=\infty$
Obviously the third set of conditions is the one applied in Section 2.4. It can be seen that all three sets are made up of single-term uniqueness conditions. For 0 , $-\infty$ and $+\infty$ may not be considered as terms for obvious reasons. This corresponds to the provisional conclusion at the end of the discussion above, suggesting that the addition of multiple-term uniqueness conditions would have led to a multitude of SQs in the optimum similarity solution.

For instance, let us consider the following pair of conditions with just one proper two-term equation (the last one):

if $x=0 \quad$ then $h=0 \quad$ and if $t=0 \quad$ then $h=a x$

i.e. a wedge-shaped initial layer of fluid with wedge angle $\tan ^{-1} a$ as seen in Fig. 2. Normalization of these conditions leads to

if $\bar{x}=0 \quad$ then $\bar{h}=0 \quad$ and if $\bar{t}=0 \quad$ then $\bar{h}=\pi_{2} \bar{x}$

where

$\pi_{2} \equiv \frac{a \hat{x}}{\hat{h}}$

Omitting the caps, which extends this normalization procedure to optimum similarity analysis, lcads to the PP

$\frac{a x}{h}$

which combined with $\bar{h}$ according to eqn. (10) forms a set of PPs for similarity which fits this model.

Next, by arranging $\pi_{1}$ and $\pi_{2}$ in two linearly independent PPs a variety of new sets may be derived that are equally suited. Take for instance

$\pi_{2}^{-1}$ and $\pi_{1}^{2} \pi_{2}^{2}$

These lead to the dependent similarity variable $\bar{h}$ and the independent similarity variable $\bar{t}$; notably

$\bar{h} \equiv h \frac{1}{a x} \quad \bar{t} \equiv t \frac{\rho g a^{2} x}{\mu}$

Consequently this modification to Jeffreys' model has the similarity solution (see also Appendix A)

$\frac{\mathrm{d} \bar{h}}{\mathrm{~d} \bar{t}}+\frac{\bar{h}^{3}}{1+\bar{h}^{2} \bar{t}}=0 \quad$ if $\bar{t}=0 \quad$ then $\bar{h}=1$

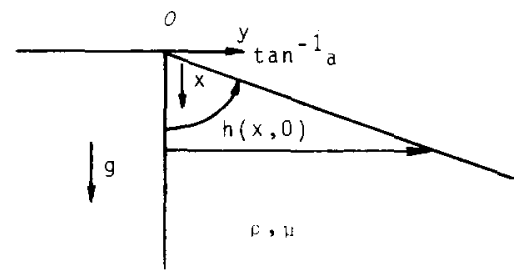

Fig. 2. A modification of Jeffreys' model. 
TABLE 2. Matrix of coefficients for the vectorial dimensional formulae for Jeffreys' model

\begin{tabular}{llrr}
\hline & $L_{x}$ & $L_{y}$ & $T$ \\
\hline$h$ & 0 & 1 & 0 \\
$x$ & 1 & 0 & 0 \\
$t$ & 0 & 0 & 1 \\
$\rho g / \mu$ & 1 & -2 & -1 \\
\hline
\end{tabular}

It can readily be solved (substituting $\bar{h}=z(\bar{t})^{-1 / 2}$ ):

$\bar{h}=\frac{(1+4 \bar{t})^{1 / 2}-1}{2 \bar{t}} \quad$ with $\bar{t}>0$

With the original quantities substituted the result is

$h=\mu \frac{\left(1+4 \rho g a^{2} t x / \mu\right)^{1 / 2}-1}{2 \rho g a t} \quad$ with $t>0$

\subsection{Discussion}

It should be mentioned here that complications may occur when applying this method to quite different models. For instance, it will be clear that a linear dependence between the $\pi_{i}$ values will lead to a reduction in the optimum set of SQs. But what should be done with integrals and functional arguments? The application to elastohydrodynamic lubrication (henceforth EHL) below may serve as an illustrative example.

In relation to dimensional analysis the following will be clear. Similarity quantities are dimensionless if and only if the basic relations are unit-free. However, all dimensionally homogeneous relations are unit-free [13]. As a consequence, dimensional analysis can only be applied to unit-free relations or, and this is the extra power of dimensional analysis, to quantities that are presumed to occur in as yet unknown unit-free relations.

This may explain why vectorial dimensional analysis lost favour. The correct arguments for the selection of the vectorial components in space follow from the constituent relations. As long as these relations remain vectorially unit-free, everything is all right. If these relations are still unknown, though, it is mere gambling. By way of an example Table 2 shows the correct way of deriving the optimum set of SQs for Jeffreys' original model by means of vectorial dimensional analysis. This could hardly have been guessed a priori, however.

\section{Elastohydrodynamic lubrication}

\subsection{The optimum similarity solution}

A more practical problem is the derivation of the smallest set of parametric SQs for the minimum film thickness model in elastohydrodynamic lubrication.
The starting point for optimum similarity analysis in EHL is the following set of relations (see ref. 14, in particular the definitions for $U_{\mathrm{s}}, R$ and $E^{\prime}$ )

$$
\begin{aligned}
& \frac{\mathrm{d}}{\mathrm{d} x}\left(\frac{h^{3}}{\mu} \frac{\mathrm{d} P}{\mathrm{~d} x}\right)=6 U_{\mathrm{s}} \frac{\mathrm{d}[(1-\theta) h]}{\mathrm{d} x} \\
& \frac{\mathrm{d} h}{\mathrm{~d} x}=\frac{x}{R}-\frac{4}{\pi E^{\prime}} \int_{-\infty}^{+\infty} \frac{P\left(x^{\prime}\right)}{x-x^{\prime}} \mathrm{d} x^{\prime}
\end{aligned}
$$

$\mu=\mu_{i} \exp (\alpha P)$

$W=\int_{-\infty}^{+\infty} P(x) \mathrm{d} x$

$h_{\min }=\min (h)$

$\theta P=0 \quad$ with $P \geqslant 0 \quad$ and $\theta \geqslant 0$

$P=0 \quad$ for $x= \pm \infty$

Equation (14) is the Reynolds equation written as a complementarity problem (in order to simulate cavitation); eqn. (15) is the elasticity equation; eqn. (16) represents the exponential viscosity-pressure relation; eqn. (17) defines the load per unit width; eqn. (18) defines the minimum film thickness and eqns. (19) and (20) define respectively the cavitation and boundary conditions that apply.

It will be convenient to arrange the quantities in five groups:

(1) The control parameters (compulsory); the order represents priority for explicit occurrence in the similarity parameters, including $W$, load per unit length; $\alpha$, the piezoviscosity coefficient; $\mu_{i}$, the viscosity at the entrance; $U_{\mathrm{s}}$, the sum velocity of the boundaries; $R$, the effective radius of curvature and $E^{\prime}$, the reduced Young's modulus. To be specific, one set of values for the control parameters defines a unique solution to the problem. The remaining parameters belong to the next group.

(2) The dependent parameters (if any); these are the parameters that do not belong to the control parameters of the preceding group, e.g. $h_{\min }$, the minimum film thickness.

(3) The independent variables (compulsory), e.g. the $x$-coordinate.

(4) The integration variables (if any), e.g. the $x^{\prime}$ coordinate. In general the independent variables of group three may function as integration variables. Sometimes, though, extra variables will be needed, as in convolution integrals.

(5) The dependent variables (compulsory), including, $P$, the pressure; $h$, the film thickness; $\mu$, the viscosity and $\theta$, the ruptured film fraction.

Next, these quantities will be arranged in the five groups of SQs as mentioned in the introduction. 
Normalization leads to eleven independent PPs of parameters. They will be arranged in groups depending on the equation that produced them, i.e. eqns. (14), (15), (16), (17) and (18) respectively:

$$
\begin{aligned}
& \pi_{1} \equiv \frac{\hat{h^{2}} \hat{P}}{\hat{x} \hat{\mu} U_{\mathrm{s}}} \quad \pi_{2} \equiv \hat{\theta} \\
& \pi_{3} \equiv \frac{\hat{h} E^{\prime}}{\hat{x} \hat{P}} \quad \pi_{4} \equiv \frac{\hat{x} E^{\prime}}{R \hat{P}} \quad \pi_{5} \equiv \frac{\hat{x}}{\hat{x}^{\prime}} \\
& \pi_{6} \equiv \frac{\hat{\mu}}{\mu_{i}} \quad \pi_{7} \equiv \alpha \hat{P} \\
& \pi_{8} \equiv \frac{W}{\hat{P} \hat{x}} \\
& \pi_{9} \equiv \frac{h_{\min }}{\hat{h}}
\end{aligned}
$$

Obviously eqns. (19) and (20) do not lead to extra PPs and neither do the integral limits, which may be written in full as $x^{\prime}= \pm \infty$ and $x= \pm \infty$. The integral expressions offer no new problems.

By arranging the results in well-chosen PPs of PPs and by subsequently omitting the caps, the following optimum set of SQs may be derived:

(1) The control similarity parameters:

$M \equiv \bar{W} \equiv W\left(\frac{1}{\mu_{i} U_{\mathrm{s}} E^{\prime} R}\right)^{1 / 2} \quad L \equiv \bar{\alpha} \equiv \alpha\left[\frac{\mu_{i} U_{\mathrm{s}}\left(E^{\prime}\right)^{3}}{R}\right]^{1 / 4}$

A load number $M$ and a piezoviscosity number $L$ have been introduced because ever since their introduction [15] (see also ref. 14) these dimensionless numbers have been generally applied in EHL.

(2) The dependent similarity parameter:

$H \equiv \bar{h}_{\min } \equiv h_{\min }\left(\frac{E^{\prime}}{\mu_{i} U_{s} R}\right)^{1 / 2}$

For reasons of convenience a minimum film thickness number $H$ has been introduced.

(3) The independent similarity variable:

$\bar{x} \equiv x\left(\frac{E^{\prime}}{\mu_{i} U_{\mathrm{s}} R^{3}}\right)^{1 / 4}$

(4) The integration similarity variable:

$\bar{x}^{\prime} \equiv x^{\prime}\left(\frac{E^{\prime}}{\mu_{i} U_{\mathrm{s}} R^{3}}\right)^{1 / 4}$

(5) The dependent similarity variables:

$$
\bar{P} \equiv P\left[\frac{R}{\mu_{i} U_{\mathrm{s}}\left(E^{\prime}\right)^{3}}\right]^{1 / 4} \quad \bar{h} \equiv h\left(\frac{E^{\prime}}{\mu_{i} U_{\mathrm{s}} R}\right)^{1 / 2}
$$

$\bar{\mu} \equiv \mu \frac{1}{\mu_{i}} \quad \bar{\theta} \equiv \theta$

The load number $M$ and the piezoviscosity number $L$ represent the new control parameters we were looking for; they stand for a reduction in the number of control parameters from six to two.

In deriving these SQs the matrix of coefficients as shown in Table 3 may be helpful. For reasons of convenience in this table the five groups of quantities have been arranged in reverse order. In this way a gaussian reduction applied to the right-hand square matrix will lead to a set of SQs for the left-hand group of quantities only. This is why, in the foregoing, arranging the quantities in a specific order has been stressed.

In doing so, the gaussian reduction will either lead to the smallest set of similarity parameters or, if no similarity parameters occur, to a reduction in the number of independent similarity variables, as has been shown for Jeffreys' model above.

The transformation of the original PPs of quantities to the final solution shown in Table 4 may still be rather toilsome. Fortunately, it can almost as well be carried out by a computer. Copies of a computer program by the name SIMANUT arc available on a diskette at a small fee. This program takes about ten seconds to analyse this EHL model on a PC; the output is illustrated in Fig. 3.

This computer program SIMANUT was originally derived just as a test for the ideas proposed in this work; in particular for the idea that similarity solutions may be derived by sheer string manipulation. Yet this quite unsophisticated program represents a very useful tool when analysing mathematical models. As to the most extensive model analysed so far: it takes about one minute to confirm the four parametric SQs for the Kirchhoff problem as derived by Tijdeman [16].

One of the main problems met when writing the computer program concerned was the introduction of logarithmic functions. Actually the primitive function for $1 / x$ should read $\log \left(x / x_{0}\right)$ with $x_{0}$ representing an

TABLE 3. Matrix of coefficients for the set of power products of parameters for EHL

\begin{tabular}{rrrrrrrrrrrrrrrr}
\hline \multicolumn{2}{r}{} & $\hat{h}$ & $\hat{\mu}$ & $\hat{\theta}$ & $\hat{x}^{\prime}$ & $\hat{x}$ & $h_{\min }$ & $W$ & $\alpha$ & $\mu_{i}$ & $U_{\mathrm{s}}$ & $R$ & $E^{\prime}$ \\
\hline$\pi_{1}$ & 1 & 2 & -1 & 0 & 0 & -1 & 0 & 0 & 0 & 0 & -1 & 0 & 0 \\
$\pi_{2}$ & 0 & 0 & 0 & 1 & 0 & 0 & 0 & 0 & 0 & 0 & 0 & 0 & 0 \\
$\pi_{3}$ & -1 & 1 & 0 & 0 & 0 & -1 & 0 & 0 & 0 & 0 & 0 & 0 & 1 \\
$\pi_{4}$ & -1 & 0 & 0 & 0 & 0 & 1 & 0 & 0 & 0 & 0 & 0 & -1 & 1 \\
$\pi_{5}$ & 0 & 0 & 0 & 0 & -1 & 1 & 0 & 0 & 0 & 0 & 0 & 0 & 0 \\
$\pi_{6}$ & 0 & 0 & 1 & 0 & 0 & 0 & 0 & 0 & 0 & -1 & 0 & 0 & 0 \\
$\pi_{7}$ & 1 & 0 & 0 & 0 & 0 & 0 & 0 & 0 & 1 & 0 & 0 & 0 & 0 \\
$\pi_{8}$ & -1 & 0 & 0 & 0 & 0 & -1 & 0 & 1 & 0 & 0 & 0 & 0 & 0 \\
$\pi_{9}$ & 0 & -1 & 0 & 0 & 0 & 0 & 1 & 0 & 0 & 0 & 0 & 0 & 0 \\
\hline
\end{tabular}


TABLE 4. Matrix of coefficients for the set of similarity quantities for EHL

\begin{tabular}{|c|c|c|c|c|c|c|c|c|c|c|c|c|c|}
\hline & $P$ & $h$ & $\mu$ & $\theta$ & $x^{\prime}$ & $x$ & $h_{\min }$ & $W$ & $\alpha$ & $\mu_{i}$ & $U_{\mathrm{s}}$ & $R$ & $E^{\prime}$ \\
\hline $\bar{P}$ & 1 & 0 & 0 & 0 & 0 & 0 & 0 & 0 & 0 & $-1 / 4$ & $-1 / 4$ & $1 / 4$ & $-3 / 4$ \\
\hline $\bar{h}$ & 0 & 1 & 0 & 0 & 0 & 0 & 0 & 0 & 0 & $-1 / 2$ & $-1 / 2$ & $-1 / 2$ & $1 / 2$ \\
\hline$\tilde{\mu}$ & 0 & 0 & 1 & 0 & 0 & 0 & 0 & 0 & 0 & -1 & 0 & 0 & 0 \\
\hline $\bar{\theta}$ & 0 & 0 & 0 & 1 & 0 & 0 & 0 & 0 & 0 & 0 & 0 & 0 & 0 \\
\hline$\tilde{\boldsymbol{x}}^{\prime}$ & 0 & 0 & 0 & 0 & 1 & 0 & 0 & 0 & 0 & $-1 / 4$ & $-1 / 4$ & $-3 / 4$ & $1 / 4$ \\
\hline $\bar{x}$ & 0 & 0 & 0 & 0 & 0 & 1 & 0 & 0 & 0 & $-1 / 4$ & $-1 / 4$ & $-3 / 4$ & $1 / 4$ \\
\hline$H \equiv \bar{h}_{\min }$ & 0 & 0 & 0 & 0 & 0 & 0 & 1 & 0 & 0 & $-1 / 2$ & $-1 / 2$ & $-1 / 2$ & $1 / 2$ \\
\hline$M \equiv \tilde{W}$ & 0 & 0 & 0 & 0 & 0 & 0 & 0 & 1 & 0 & $-1 / 2$ & $-1 / 2$ & $-1 / 2$ & $-1 / 2$ \\
\hline$L \equiv \bar{\alpha}$ & 0 & 0 & 0 & 0 & 0 & 0 & 0 & 0 & 1 & $1 / 4$ & $1 / 4$ & $-1 / 4$ & $3 / 4$ \\
\hline
\end{tabular}

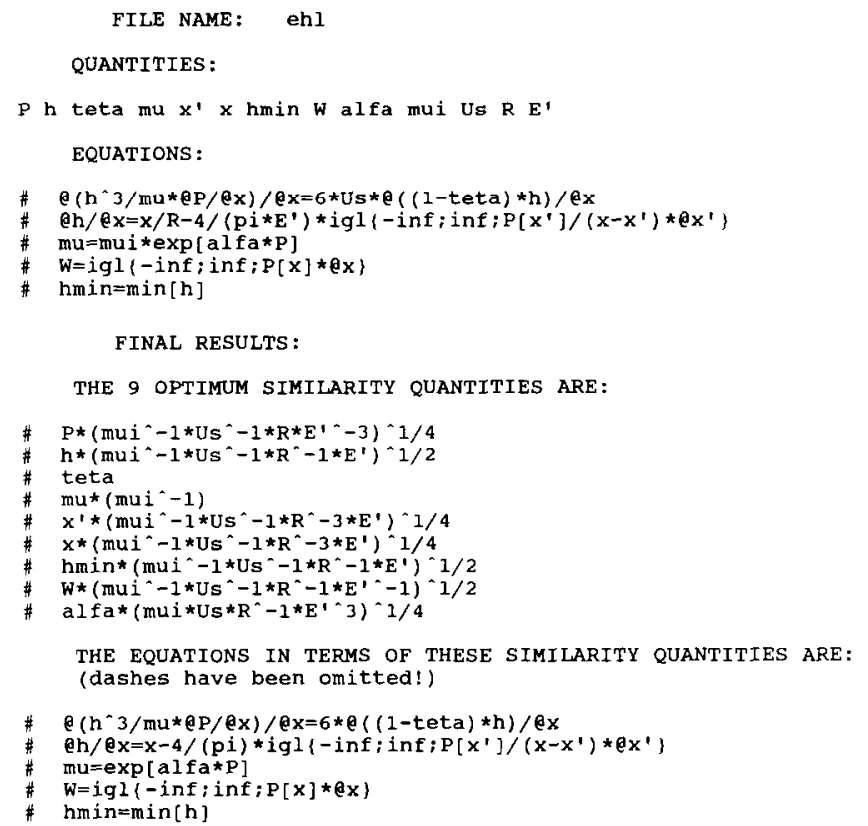

Fig. 3. Output of the computer program SIMANUT for the EHL model.

arbitrary constant, since the alternative, $\log (x)+C$, may lead to complications. For the same reason $\log (A / B)$ is to be preferred rather than $\log (A)-\log (B)$ and so on.

\subsection{Survey diagrams}

In this way a proof has been given that indeed the minimum film thickness in EHL may be considered as depending on two control parameters only. As a consequence the extra control parameter defined [10] by

$\frac{E^{\prime} R}{\mu_{i} U_{s}}$

although dimensionless, is redundant and the minimum film thickness number $H$ depends exclusively on the load number $M$ and on the piezoviscosity number $L$; i.e.

$H=H(M, L)$
Calculated data and measurements, therefore, may readily be plotted into just one diagram and curve fitting can easily be performed. In addition, the following four asymptotic solutions that apply will prove to be very useful.

(1) The rigid-isoviscous (index RI) asymptotic solution may be derived by eliminating both $\alpha$ and $E^{\prime}$ from these three similarity parameters, i.e.

$H_{\mathrm{RI}}=C_{\mathrm{RI}} M^{-1} \quad$ for $M \longrightarrow 0$

Martin [17] has shown that $C_{\mathrm{RI}}=2.44748 \ldots$

(2) The rigid-piezoviscous (index RP) asymptotic solution may be derived by eliminating both $W$ and $E^{\prime}$, i.e.

$H_{\mathrm{RP}}=C_{\mathrm{RP}} L^{-2 / 3} \quad$ for $L \longrightarrow \infty$

Grubin [18] has shown that $C_{\mathrm{RP}}=1.04970 \ldots$

(3) The elastic-isoviscous (index EI) asymptotic solution may be derived by climinating $\alpha$, i.e.

$H_{\mathrm{F}, \mathrm{I}}=C_{\mathrm{EI}} M^{\alpha} \quad$ for $M \longrightarrow \infty \quad$ and $L=0$

We have shown [19] that $\alpha=-\frac{1}{5}$ and $C_{E I}=2.04594 \ldots$

(4) The elastic-piezoviscous (index EP) asymptotic solution may be expected to look like

$H_{\mathrm{EP}}=C_{\mathrm{EP}} M^{\beta} L^{\gamma} \quad$ for $M \longrightarrow \infty$

Crook [20] shows that $\beta=-\frac{1}{8}, \quad \gamma=\frac{3}{4}$ and $C_{\mathrm{EP}}=$ $0.856526 \ldots$

For more information about the derivation of these asymptotic solutions see ref. 19 .

Figure 4 shows a survey of the complete solution for the EHL at line contact that has been based on these four asymptotic solutions. This diagram has been drawn by applying the curve-fit

$$
\begin{aligned}
H= & \left\{\left[\left(\left\{1-\exp \left[-\left(\frac{H_{\mathrm{RP}}}{H_{\mathrm{EP}}}\right)^{5 / 2}\right]\right\} H_{\mathrm{EP}}^{5 / 2}\right)^{8 / 15}\right.\right. \\
& \left.\left.+H_{\mathrm{EI}}^{4 / 3}\right]^{7 / 4}+H_{\mathrm{RI}}{ }^{7 / 3}\right\}^{3 / 7}
\end{aligned}
$$




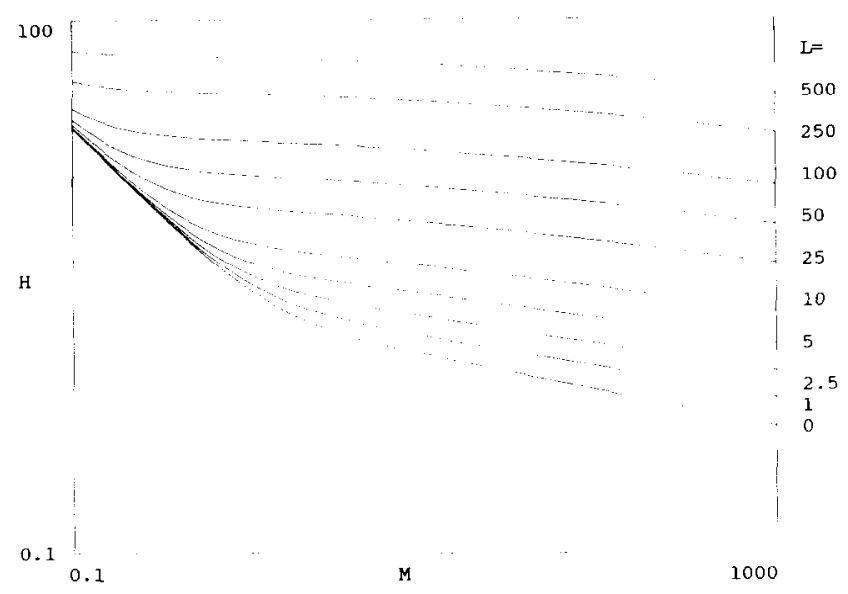

Fig. 4. Survey diagram for EHL; logarithmic axes.

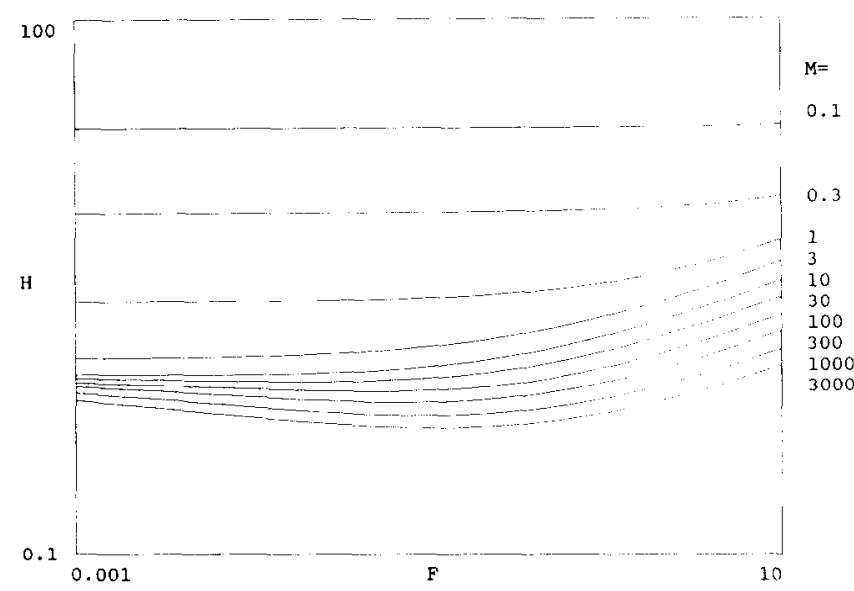

Fig. 5. The effect of flexibility on film thickness; logarithmic axes.

The quite accurate numerical solutions in the transition areas between the asymptotic solutions calculated by Venner [21] have been compared with our curve-fit [19]. This confirms the high quality of the curve-fit, in particular in comparison with our original diagram [22], but also in comparison with the diagrams introduced by Lubrecht [23] and Venner [21].

By rearranging the order of priority of the control parameters for explicit occurence in the similarity parameters a great number of alternative survey diagrams may be generated. One of these diagrams, represented by Fig. 5, is of particular interest. It shows the effect of the flexibility on the minimum film thickness. This time the symbols applied are defined by

$$
\begin{aligned}
& H \equiv h_{\min }\left(\frac{1}{\alpha^{2} \mu_{i}^{2} U_{\mathrm{s}}^{2} R}\right)^{1 / 3} \quad M \equiv W\left(\frac{\alpha^{2}}{\mu_{i} U_{\mathrm{s}} R^{2}}\right)^{1 / 3} \\
& F \equiv \frac{1}{E^{\prime}}\left(\frac{R}{\alpha^{4} \mu_{i} U_{\mathrm{s}}}\right)^{1 / 3}
\end{aligned}
$$

Where $F$ represents a flexibility number. The diagram is based on eqn. (21).

As might have been expected, the film thickness generally increases with increasing flexibility. However, quite surprisingly in this diagram a small area occurs where the film thickness decreases with increasing flexibility. Incidentally this area happens to represent most of the applications. A second computer program by the name of EHLUT, this time for calculating the minimum film thickness in EHL based on the curvefit presented above, is also available on a diskette at a small fee.

Of course, when applying optimal similarity analysis to dissimilar models complications may arise. However, the analysis of special problems in EHL does not lead to complications. Of interest are the applications listed with the computer program SIMANUT mentioned above in Section 4.1.

\section{Discussion and conclusions}

The new technique for obtaining similarity solutions as introduced in this paper is both simple and straightforward. Therefore it is a very attractive tool for engineers applying mathematical analysis.

Essentially, this optimum similarity method is an extension of the well-known and generally applied technique of normalizing equations, although the method is much more powerful, since optimum similarity solutions will invariably be derived. Moreover, the application is simple. For complicated models, the computer program SIMANUT, mentioned above may be very helpful.

Of course, a drawback as compared with dimensional analysis is that the complete mathematical formulation has to be known "a priori". However, the advantage is that an optimum similarity solution will be obtained, whether the relations are unit-free or not.

Apparently a paradox is presented when comparing Buckingham's pi-theorem with the derivation of similarity quantities as presented in this paper. This is because the pi-theorem refers to fundamental dimensional quantities $[24,25]$, whereas actually the optimum set of similarity quantities depends on the number of terms, equations etc. involved, which has nothing at all to do with dimensions.

This paradox may be overcome by noticing that the number of similarity quantities, as derived by applying dimensional analysis or by applying the pi-theorem, actually may be any number in between the number of elements in the optimum set of similarity quantities and the number of quantities appearing in the set of relations under consideration. The result depends on the kind of dimensional analysis applied. For instance, 
it will depend on whether there is to be discrimination between two or even three spatial components, and on whether there is to be discrimination between gravitational mass and inertial mass in a newtonian world.

As a consequence, our final conclusion needs to be that these results do show that physical properties are out of place in similarity analysis. This may lead to the challenging conclusion that similarity analysis has nothing whatsoever to do with physics. In fact similarity analysis is limited to sheer string manipulation. However, the reader should be aware that mathematical analysis in general is definitely more than just syntax.

\section{References}

1 W. F. Ames, Nonlinear Partial Differential Equations in Engineering, Vol. 1; Mathematics in Science and Engineering, Vol. 18, Academic Press, London, 1965.

2 P. W. Bridgeman, Dimensional Analysis, 2nd edn., Yale University Press, 1931.

3 H. E. Huntley, Dimensional Analysis, Macdonald, London, 1952.

4 J. Palacios, Dimensional Analysis, Macmillan, London, 1964.

5 A. Vaschy, Sur les lois de similitude en physique, Ann. Telegr., 19 (1892) 25-28.

6 E. Buckingham, On physical similar systems, Phys. Rev., 4 (1914) 345-376.

7 E. Buckingham, Model experiments and the forms of empirical equations, Trans. ASME, 37 (1915) 263-296.

8 H. L. Langhaar, Dimensional Analysis and Theory of Models, Wiley, New York, 1951.

9 H. Schlichting, Grenzschicht-Theorie, G. Braun, Karlsruhe, 1951.

10 D. Dowson, Elastohydrodynamic lubrication: an introduction and a review of theoretical studies, Proc. Inst. Mech. Eng., London, Part 3B, 180 (1965) 7-16.

11 A. E. Ruark, Inspectional analysis: a method which supplements dimensional analysis, J. Elisha Mitchell Sci. Soc., 51 (1935) 127-133.

$12 \mathrm{H}$. Jeffreys, The drainage of a vertical plate, Proc. Cambridge Philos. Soc., 26 (1930) 730-731.

13 G. Birkhoff, Hydrodynamics, Princeton University Press, Princeton, NJ, 1960

14 D. Dowson and G. R. Higginson, Elasto-hydrodynamic Lubrication, Pergamon, Oxford, 1966.

15 O. J. Koets, A Survey of the Isothermal Theory of Elastohydrodynamic Lubrication, Delft University Technology Report, 1962.

$16 \mathrm{H}$. Tijdeman, On the propagation of sound waves in cylindrical tubes, $I$. Sound Vib., 39 (1975) 1-33.

17 H. M. Martin, Lubrication of gear teeth, Engineening, 11 (1916) 119-121.

18 A. N. Grubin, Fundamentals of the hydrodynamic theory of lubrication of heavily loaded cylindrical surfaces, in $\mathrm{Kh}$. F. Ketova (ed.), Investigation of the Contact of Machine Components, Moscow: TsNIITMASh, Book 30, 1949. (D.S.I.R., London, Translation 337, pp. 115-166).

$19 \mathrm{H}$. Moes, A survey of EHL solutions, to be published in 1993.

20 A. W. Crook, The lubrication of rollers - II. Film thickness with relation to viscosity and speed, Philos. Trans. $R$. Soc. London, Ser. A, 254 (1961) 223-258.
21 C. H. Venner, Multilevel solution of the EHL line and point contact problems, Ph.D. Thesis, University of Twente, Enschede, 1991.

$22 \mathrm{H}$. Moes, Discussion on a paper by D. Dowson, Proc., Inst. Mech. Eng., London, Part 3B, 180 (1965) 244-245.

23 A. A. Lubrecht, The numerical solution of the elastohydrodynamically lubricated line and point contact problem, using multigrid techniques, Ph.D. Thesis, University of Twente, Enschede, 1987.

24 A. G. Hansen, Similarity Analysis of Boundary Value Problems in Engineering, Prentice Hall, Englewood Cliffs, NJ, 1964.

25 R. Saint-Guilhem, Les Principes de l'Analyse Dimensionelle, Gauthier-Villars, Paris, 1962.

\section{Appendix A: Transformation of the variables}

The transformation to new independent variables in differential equations is not always easily performed. The following equation may be helpful:

$\frac{\partial Q_{i}}{\partial Q_{j}}=\frac{Q_{i}}{\bar{Q}_{i} Q_{j}}\left(\sum_{k-d+1}^{n} \alpha_{k j} \bar{Q}_{k} \frac{\partial \bar{Q}_{i}}{\partial \bar{Q}_{k}}-\alpha_{i j} \bar{Q}_{i}\right)$

where $i=1, \ldots, d$ and $j=d+1, \ldots, n$. In this equation $d$ represents the number of dependent variables, which corresponds to the number of dependent similarity variables, and $n-d$ represents the number of independent similarity variables. Furthermore, $\alpha_{i j}$ represents the power of the independent variable $Q_{j}$ in the independent similarity variable $\bar{Q}_{i}$ with $i=d+1, \ldots, n$; see also eqn. (1).

For instance, eqn. (9) originates from substituting for the partial derivatives in the eqn. (2):

$\frac{\partial h}{\partial t}=\frac{h}{\bar{h} t}\left(\bar{x} \frac{\mathrm{d} \bar{h}}{\mathrm{~d} \bar{x}}-\bar{h}\right) \quad$ and $\quad \frac{\partial h}{\partial x}=\frac{h}{\bar{h} x}\left(\bar{x} \frac{\mathrm{d} \bar{h}}{\mathrm{~d} \bar{x}}\right)$

and eqn. (11) originates from substituting for the partial derivatives in eqn. (2):

$\frac{\partial h}{\partial t}=\frac{h}{\bar{h} t}\left(\frac{1}{2} \bar{h}\right) \quad$ and $\quad \frac{\partial h}{\partial x}=\frac{h}{\bar{h} x}\left(-\frac{1}{2} \bar{h}\right)$

and eqn. (13) originates from substituting for the partial derivatives in eqn. (2):

$\frac{\partial h}{\partial t}=\frac{h}{\bar{h} t}\left(\bar{t} \frac{\mathrm{d} \bar{h}}{\mathrm{~d} \bar{t}}\right) \quad$ and $\quad \frac{\partial h}{\partial x}=\frac{h}{\bar{h} x}\left(\bar{t} \frac{\mathrm{d} \bar{h}}{\mathrm{~d} \bar{t}}+\bar{h}\right)$

\section{Appendix B: Nomenclature}

$E^{\prime} \quad$ reduced Young's modulus, $\mathrm{FL}^{-2}$

$F \quad$ flexibility number

$g \quad$ acceleration due to gravity, $\mathrm{LT}^{-2}$

$h$ thickness of film or fluid layer, $\mathrm{L}$

$h_{\min } \quad$ minimum film thickness, $\mathrm{L}$

$H \quad$ minimum film thickness number 
$L \quad$ piezoviscosity number

$M \quad$ load number

$P \quad$ pressure, $\mathrm{FL}^{-2}$

$Q_{i} \quad$ quantity

$\bar{Q}_{i} \quad$ characteristic similarity quantity for the quantity $Q_{i}$

$R \quad$ effective radius of curvature, $\mathrm{L}$

$t \quad$ time, $\mathrm{T}$

$U_{\mathrm{s}} \quad$ sum of the tangential surface velocities, $\mathrm{L} / \mathrm{T}$

$W \quad$ load per unit length, $\mathrm{F} / \mathrm{L}$

$x, x^{\prime}, y$ coordinates, $\mathrm{L}$

Greek symbols

$\alpha \quad$ piezoviscosity coefficient, $\mathrm{L}^{2} / \mathrm{F}$ $\theta \quad$ ruptured film fraction

$\mu \quad$ fluid viscosity, $\mathrm{FTL}^{-2}$

$\mu_{i} \quad$ viscosity at entrance, FTL $^{-2}$

$\pi_{i} \quad$ dimensionless number

$\rho \quad$ fluid density, $\mathrm{FT}^{2} \mathrm{~L}^{-4}$

Abbreviations

EHL Elasto hydrodynamic lubrication

EI Elastic-isoviscous

EP Elastic-piezoviscous

PP Power product of quantities

RI Rigid-isoviscous

RP Rigid-piezoviscous

SQ Similarity quantity 